\title{
Quantitative Comparison of Agile Modulation Techniques for Cognitive Radio Transceivers
}

\author{
Rakesh Rajbanshi \\ Qi Chen \\ Alexander M. Wyglinski \\ Gary J. Minden \\ Joseph B. Evans \\ Information and Telecommunication Technology Center \\ The University of Kansas, Lawrence, KS 66045 \\ Email: \{rajbansh, chenqi, alexw, gminden, evans\}@ittc.ku.edu
}

\begin{abstract}
In this paper, we present a quantitative comparison of two agile modulation techniques employed by cognitive radio transceivers operating in a dynamic spectrum access (DSA) network. One of the modulation technique is non-contiguous orthogonal frequency division multiplexing (NC-OFDM), which is designed to avoid interference with the transmissions of incumbent users by deactivating subcarriers within their vicinity. The other modulation technique under study is a variant of multicarrier code division multiple access (MC-CDMA). Although several studies comparing conventional OFDM and MC-CDMA has been conducted in literature to justify robust error performance of $\mathrm{MC}$ CDMA, a quantitative performance evaluation of these schemes has not been performed when employed in a DSA network. Due to deactivated subcarriers in DSA networks, in this paper we showed their performance can be significantly different from the conventional setup. Analytical expressions for the error probability of an NC-OFDM transceiver have been derived and compared with computer simulation results. The results show that the error robustness of NC-OFDM is relatively constant regardless of the number of deactivated subcarriers, unlike MC-CDMA transmissions, whose error performance degrades with an increase in deactivated subcarriers.
\end{abstract}

\section{Introduction}

To meet the growing demand for transmission bandwidth required by current and future wireless services and applications, the Federal Communications Commission (FCC) has begun work on defining a new spectrum allocation concept called dynamic spectrum access (DSA) [1]. With advances in software-defined radio (SDR) technology, where the baseband processing is performed entirely in software, current radio transceivers are sufficiently agile to operate in a DSA networking environment due to their ease and speed of programming baseband operations. SDR units that can rapidly and autonomously reconfigure operating parameters due to changing requirements and conditions ${ }^{1}$ are known as cognitive radios [2]. With recent developments in cognitive radio technology, it is now possible for these systems to simultaneously respect the rights of incumbent license holders while

This work was supported by NSF grants ANI-0230786 and ANI0335272 .

${ }^{1}$ These requirements and conditions can be at the physical, network, and/or application layers of the system. providing additional flexibility and access to spectrum.

The choice of physical layer transmission technique is a very important design decision when implementing a cognitive radio. To support high data-rate transmissions, the technique should be sufficiently agile to enable users to use a large bandwidth without interfering with incumbent users. Orthogonal frequency division multiplexing (OFDM) and multicarrier code division multiple access (MC-CDMA) are two high-speed modulation techniques employed in conventional transmission systems. OFDM has been shown to be an effective transmission technique for high speed data communications. On the other hand, MC-CDMA is capable of mitigating the effects of multiuser interference. Several studies have justified superior error performance of MC-CDMA system over OFDM system [3,4]. In DSA network, subcarriers belonging to unlicensed device, i.e. secondary user, that are located in the vicinity of an incumbent user, i.e. primary user, are deactivated to avoid interference. These variants are called non-contiguous OFDM (NC-OFDM) and non-contiguous MC-CDMA (NCMC-CDMA).

In this paper, we conduct a quantitative comparison of NC-OFDM and NC-MC-CDMA transmission techniques within the context of a DSA network ${ }^{2}$. The analytical expressions for the probability of error of an NC-OFDM transceiver is presented and validated using computer simulations $^{3}$. Then, we compare NC-OFDM and NC-MCCDMA, in terms of error robustness. The paper is organized as follows: In Section 2, brief introduction to the NC-OFDM and NC-MC-CDMA transmission techniques are presented. In Section 3, a frequency selective Rayleigh fading channel model for BER performance analysis is presented. Theoretical SNR analysis of the NC-OFDM system is presented in Section 4. Finally, Section 5 presents the BER performance comparison between NC-OFDM and NC-MC-CDMA techniques for data transmissions over noncontiguous spectrum.

\footnotetext{
${ }^{2}$ This work builds upon the authors' previous paper, presented at the 2nd Annual International Wireless Internet Conference (WICON) - Workshop on Technology and Policy for Accessing Spectrum (TAPAS) [5], by providing an analytical analysis of the error performance of the NC-OFDM system.

${ }^{3}$ We assume that the secondary user has perfect knowledge about the spectrum occupancy.
} 


\section{System Model}

When portions of the target licensed spectrum are occupied by incumbent users, multicarrier techniques can provide the necessary agile spectrum usage [6,7]. Multicarrierbased transceivers can deactivate, i.e. "null", subcarriers that could potentially interfere with other users, while also providing high data rates at an acceptable level of error robustness $[8,9]$. Both OFDM and MC-CDMA are popular multicarrier transmission techniques. In this section, we present a brief overview of NC-OFDM and NC-MC-CDMA transmission frameworks ${ }^{4}$.

\subsection{NC-OFDM Framework}

In an NC-OFDM transceiver, a high speed data stream, $x(n)$, is modulated using $M$-ary phase shift keying (MPSK) or quadrature amplitude modulation (MQAM), and split into $N$ slower data streams using a serial-to-parallel (S/P) converter. Unlike conventional OFDM, not all of the subcarriers are active ${ }^{5}$, and thus do not transmit any data. The inverse fast Fourier transform (IFFT) is then applied to these modulated subcarrier signals, followed by the parallelto-serial $(\mathrm{P} / \mathrm{S})$ conversion. The baseband NC-OFDM signal, $s(n)$, is then passed through the transmitter radio frequency (RF) chain, which amplifies the signal and upconverts it to the desired center frequency.

The receiver performs the reverse operation of the transmitter, mixing the RF signal to baseband for processing, yielding the signal $r(n)$. Then, the signal is converted into parallel streams using $\mathrm{S} / \mathrm{P}$ converter, and the fast Fourier transform (FFT) is applied to transform the time domain data into the frequency domain. After compensating distortion introduced by the channel, the data in the active subcarriers is multiplexed using a $\mathrm{P} / \mathrm{S}$ converter, and demodulated into a reconstructed version of the original highspeed input, $\hat{x}(n)$.

\subsection{NC-MC-CDMA Framework}

The structure of MC-CDMA was devised in order to overcome the high sampling rates required by direct sequence CDMA (DS-CDMA) transmission, where spreading is performed in the time domain. This high sampling rate makes DS-CDMA very susceptible to performance degradation caused by multipath propagation [12]. To avoid any interference to existing transmissions, subcarriers that interfere with occupied portions of spectrum are deactivated, in much the same way as is done in NC-OFDM.

As with the NC-OFDM transceiver, the NC-MC-CDNA system begins by taking the high data rate input, $x(n)$, and feeding it into an MPSK or MQAM modulator prior to serial-to-parallel (S/P) conversion into $L$ streams. Each of these streams has a data rate less than $x(n)$ by a factor of $L$.

\footnotetext{
${ }^{4}$ For details on the NC-OFDM and NC-MC-CDMA frameworks, please refer to the authors' previous work in this area [5].

${ }^{5}$ Active subcarriers are located in the unoccupied spectrum bands, which are determined by dynamic spectrum sensing and channel estimation techniques $[10,11]$.
}

Following the $\mathrm{S} / \mathrm{P}$ conversion, each stream is replicated into $N$ parallel copies ${ }^{6}$, with copy $m$ of stream $k$ being multiplied by chip $m$ of spreading code $C_{k}$, for $k=0, \ldots, L-1$ and $m=0, \ldots, N-1$ [4]. This is referred to as spreading in the frequency domain. Note that all the spreading codes used must be orthogonal with each other. After the frequency domain spreading, copy $m$ of all the streams are added together, for $m=0, \ldots, N-1$, yielding $N$ subcarrier inputs to the IFFT block, which converts these subcarriers into the time domain. The resulting normalized complex envelope of an MPSK-modulated MC-CDMA signal is given as,

$$
s(n)=\frac{1}{\sqrt{N}} \sum_{k=0}^{L-1} \sum_{m=0}^{N-1} b_{k} C_{k, m} e^{j 2 \pi m n / T},
$$

where $b_{k}$ is the MPSK-modulated symbol from $k^{\text {th }}$ stream, and $C_{k, m}$ is chip $m$ of spreading sequence $k$. Following the $\mathrm{P} / \mathrm{S}$ conversion, the baseband NC-MC-CDMA signal, $s(n)$, is then passed through the transmitter RF chain, which amplifies the signal and upconverts it to the desired center frequency.

The receiver performs the reverse operation of the transmitter, where the received baseband signal $r(n)$ undergoes $\mathrm{S} / \mathrm{P}$ conversion, time-to-frequency conversion via FFT, and equalization. Each of the equalizers outputs are then replicated into $L$ parallel copies, with each copy allocated to one of $L$ streams, where despreading is performed using $C_{k}$, for $k=0, \ldots, L-1$. An integrate-and-dump procedure is then performed per stream, followed by $\mathrm{P} / \mathrm{S}$ conversion and demodulation. This results in a reconstructed version of the original high data rate input signal, $\hat{x}(n)$.

In order to compare with NC-OFDM, it is necessary that both implementations employ identical data rates. Therefore, the number of streams, $L$, must also be reduced. Note that when all of the subcarriers are active, $L=N$.

\section{Channel Model}

For both the NC-OFDM and NC-MC-CDMA systems, we assume the maximum delay of the multipath channel is shorter than the cyclic prefix. Moreover, the transmitter and the receiver are perfectly synchronized with each other and the channel is pseudo-stationary.

With these assumptions, the $i^{\text {th }}$ received symbol can be described as follows:

$$
Y_{i}=X_{i} \cdot H_{i}+\tilde{n_{i}}
$$

where $Y_{i}$ represents $N$ received data symbols, $X_{i}$ represents $N$ transmitted data symbols, $H_{i}=\mathrm{FFT}\left(h_{i}\right)$ is the frequency response of the channel, $h_{i}$ is the impulse response of the channel padded with zeros to obtain length of $N$, and $\tilde{n}_{i}=\operatorname{FFT}\left(n_{i}\right)$, with $n_{i}$ representing zero-mean complex Gaussian independent random variables.

We use a general equivalent baseband multipath channel model [13]. The channel consisting of $M$ multipath compo-

\footnotetext{
${ }^{6}$ The data rate of the stream and its copies are identical.
} 
nents has the form of:

$$
h(\tau)=\sum_{m=0}^{M-1} a_{m} \delta\left(\tau-\tau_{m}\right)
$$

where $a_{m}$ is a zero-mean complex Gaussian independent random variable and $\tau_{m}$ is the delay of the $m^{\text {th }}$ path.

We assume an exponential power delay profile given by:

$$
\begin{aligned}
E\left[h(\tau) h^{*}(\tau)\right] & =E\left[a_{m} a_{m}^{*}\right] \\
& =C e^{-\tau / \tau_{\mathrm{rms}}}, 0<\tau<\tau_{\max }
\end{aligned}
$$

where $\tau_{\text {rms }}$ is the rms delay spread, $\tau_{\max }$ is the maximum excess delay, and $C$ is the normalization constant which makes the total multipath power equal to 1, i.e.,

$$
\sum_{m=0}^{M-1} E\left[a_{m} a_{m}^{*}\right]=1 .
$$

\section{Signal-to-Noise Ratio Analysis}

The signal-to-noise ratio (SNR) is defined as the ratio of the desired signal power to the noise power [13]. The SNR indicates reliability of transmission link between the transmitter and receiver, and is accepted as a standard measure of signal quality.

Assuming a wide sense stationary uncorrelated scattering (WSSUS) channel [14], the SNR of the received signal in Eq. (2) is given by:

$$
\gamma_{i}^{f}=\frac{\left|X_{i} \cdot H_{i}\right|^{2}}{\left|\tilde{n}_{i}\right|^{2}}=\frac{\left|X_{i}\right|^{2} \cdot\left|H_{i}\right|^{2}}{\left|\tilde{n}_{i}\right|^{2}} .
$$

Therefore, the mean SNR can be given by [15]:

$$
E\left(\gamma_{i}^{f}\right)=\frac{E\left(\left|X_{i}\right|^{2} \cdot\left|H_{i}\right|^{2}\right)}{E\left(\left|\tilde{n}_{i}\right|^{2}\right)}=\frac{E\left(\left|X_{i}\right|^{2}\right) \cdot E\left(\left|H_{i}\right|^{2}\right)}{E\left(\left|\tilde{n_{i}}\right|^{2}\right)}
$$

where $E(\cdot)$ denotes an expectation operator.

In the following two subsections, we present the SNR analysis for the NC-OFDM system over additive white Gaussian noise (AWGN) and Rayleigh fading channels.

\subsection{AWGN Channel}

Consider an AWGN channel with noise spectral density $N_{0}$ and bandwidth $B$, the noise power is given by:

$$
E\left(\left|\tilde{n}_{i}\right|^{2}\right)=\sigma_{N}^{2}=N_{0} B
$$

while the SNR is given by:

$$
\gamma_{1}=10 \log _{10}\left(\frac{E\left(\left|X_{i}\right|^{2}\right)}{\sigma_{N}^{2}}\right)=10 \log _{10}\left(\frac{E\left(\left|X_{i}\right|^{2}\right)}{N_{0} B}\right) .
$$

Suppose the incumbent spectral occupancy ${ }^{7}$ (ISO) is $\alpha$, then the total available bandwidth would be $(1-\alpha) B$. Since the channel response is assumed to be flat, the signal power would remain constant, irrespective of the available bandwidth. However, the effective noise power would be:

$$
\sigma_{N}^{2}=N_{0}(1-\alpha) B
$$

\footnotetext{
${ }^{7}$ Incumbent spectral occupancy (ISO) is defined as the fraction of the intended transmission bandwidth occupied by incumbent user transmissions.
}

with the SNR given by:

$$
\gamma_{2}=10 \log _{10}\left(\frac{E\left(\left|X_{i}\right|^{2}\right)}{\sigma_{N}^{2}}\right)=10 \log _{10}\left(\frac{E\left(\left|X_{i}\right|^{2}\right)}{N_{0}(1-\alpha) B}\right) .
$$

Therefore, the SNR gain is:

$$
\mathrm{SNR}_{\text {gain }}=-10 \log _{10}(1-\alpha) \text {. }
$$

However, the total throughput would also be reduced to $(1-\alpha) N R_{b}$, where $R_{b}$ represents the bit rate over an individual subcarrier.

\subsection{Rayleigh Fading Channel}

Suppose we consider a frequency non-selective slow fading channel, i.e. flat channel response, where the channel magnitude response $E\left(\left|H_{i}\right|^{2}\right)$ is flat over the spectrum band. The deactivation of subcarriers due to incumbent users will result in a non-zero ISO. This would also filter out a portion of the channel magnitude response, which results in an increase in the magnitude of $E\left(\left|H_{i}\right|^{2}\right)$. As a result, the SNR gain is given by:

$$
\begin{aligned}
\mathrm{SNR}_{\text {gain }} & =10 \log _{10}\left(\frac{E\left(\left|X_{i}\right|^{2}\right) \cdot E\left(\left|H_{i}\right|^{2}\right) /(1-\alpha)}{N_{0}(1-\alpha) B}\right) \\
& -10 \log _{10}\left(\frac{E\left(\left|X_{i}\right|^{2}\right) \cdot E\left(\left|H_{i}\right|^{2}\right)}{N_{0} B}\right) \\
& =-10 \log _{10}(1-\alpha)^{2} .
\end{aligned}
$$

In case of frequency selective multipath channel, the channel magnitude response $E\left(\left|H_{i}\right|^{2}\right)$ is not flat over the spectrum. Thus, deactivating a portion of the spectrum would also flatten a portion of the channel magnitude response, which results in a random increase in the magnitude of $E\left(\left|H_{i}\right|^{2}\right)$. Therefore, the SNR gain would not be linear as in the case with a flat AWGN channel.

\subsection{NC-MC-CDMA Orthogonality Analysis}

Since the subcarriers corresponding to the incumbent user transmissions are deactivated to avoid any interference to the existing users, the information over these subcarriers are lost. This causes the loss of orthogonality between the spreading codes and raises the irreducible error floor in NCMC-CDMA system. In this work, MC-CDMA framework is modified just to avoid any interference to the primary users and for the fair comparison, the subcarriers are turned off in the same way as in NC-OFDM framework ${ }^{8}$.

For analysis, we consider that subcarriers are perfectly orthogonal and cyclic prefix is sufficiently longer than channel delay spread. Therefore, loss of orthogonality of the spreading codes, due to deactivating subcarriers, would be a major contributing factor for the interference on NC-MC-CDMA symbols.

Intuitively, loss of information in spreading codes due to nulling of the subcarriers causes the loss of orthogonality,

\footnotetext{
${ }^{8}$ The performance of the NC-MC-CDMA can be improved with adaptive code selection process to minimize the effect of loss of information. However, the process would be highly dependent upon the number and locations of nulled subcarriers, and therefore not considered in this work.
} 
leading to worse BER performance as compared to that in NC-OFDM system in both AWGN and Rayleigh fading channel.

\section{System Performance}

\subsection{Simulation Setup}

For the simulations of the NC-OFDM and NC-MC-CDMA transceivers, $N=128$ BPSK-modulated subcarriers were employed. Comparisons were performed when 0\%, $5 \%$, $10 \%, 15 \%, 20 \%$, and $25 \%$ of the $N$ total number of subcarriers were deactivated, modeling the effects of incumbent user spectral occupancy within the transmission bandwidth. A three-path Rayleigh channel model with an exponential power delay profile was used [14], where each of the multipath components is an independent and identically distributed (i.i.d.) zero-mean complex Gaussian random variable. The cyclic prefix length for both transceivers was three samples long (2.5\% of the symbol). Channel distortion compensation was performed using per tone equalization for each subcarrier [9]. The transceivers for both systems were assumed to be perfectly synchronized, the channel fading was considered to be pseudo-stationary, i.e., do not vary over a long period of time, and no coding was performed for the purpose of straightforward comparison. For each SNR point, the simulations continued until 100 bit errors were recorded, and each BER point was averaged over 100 channel realizations.

\subsection{BER Performance Analysis}

The BER results for an NC-OFDM and an NC-MC-CDMA transceiver operating in an AWGN channel for different percentages of deactivated subcarriers are shown in Fig. 1(a). When $0 \%$ of the subcarriers are deactivated, both transceivers have the exact same performance when operating in the AWGN channel. Moreover, their curves in this case also match the theoretical BER curve for a single carrier BPSK-modulated transceiver operating in an AWGN channel, which is true for both transceivers. On the other hand, when the percentage of deactivated subcarriers increases, the performance of the two transceivers begins to differ. The BER performance of the NC-OFDM transceiver slightly improves relative to the $0 \%$ curve due to fewer contributions of subcarrier noise ${ }^{9}$, as shown in Fig. 2(a). However, the BER performance of the NC-MC-CDMA degrades as the number of deactivated subcarriers increases. This is due to the fact that the subcarriers are dependent on each other since the information from the original $L$ streams have been spreaded across them all. Thus, the deactivation of a subcarrier will result in the loss of some information, which would have been used at the receiver to reconstruct the original streams. The degradation in BER performance of NC-MC-CDMA systems is shown in Fig. 3.

Given a three-path Rayleigh multipath channel for $\tau_{r m s} / T_{s}=0.1$ were studied, where $T_{s}$ is the NC-OFDM

\footnotetext{
${ }^{9}$ Since the channel is AWGN, the channel gain is flat and the effect of the $E\left(\left|H_{i}\right|\right)$ is non-existent.
}

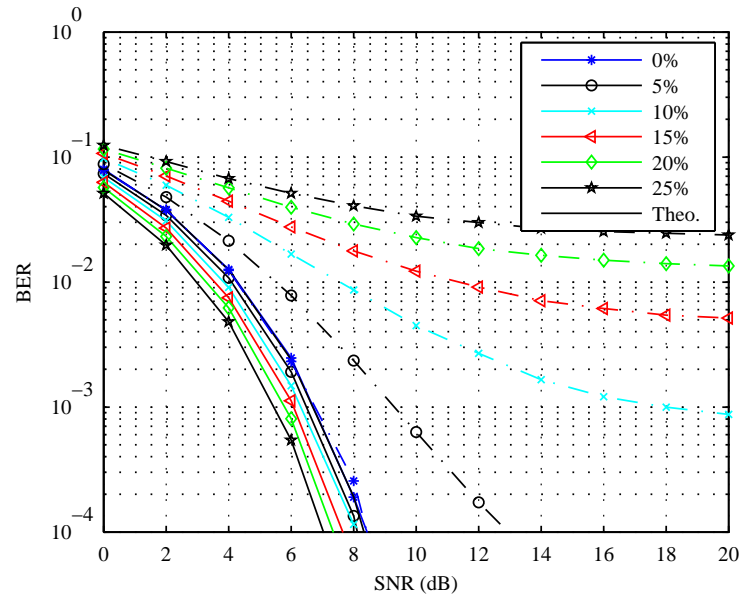

(a) AWGN channel.

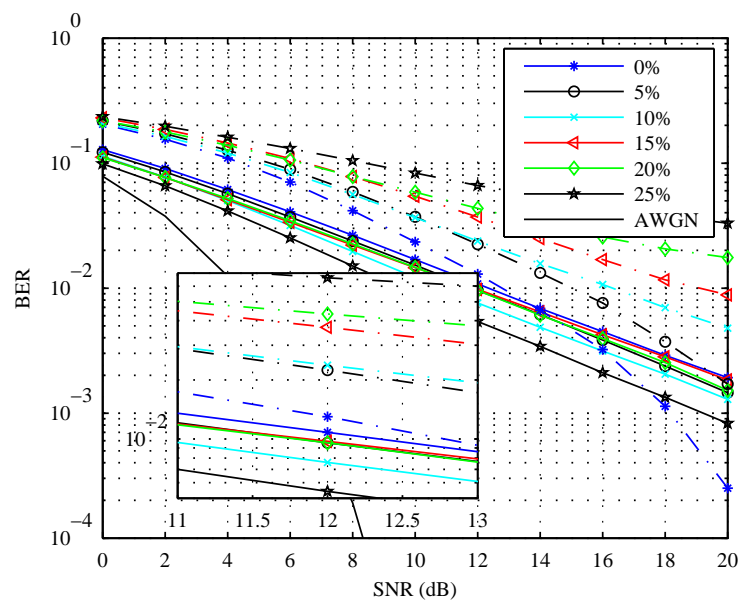

(b) 3-path Rayleigh channel.

Fig. 1 BER performance of NC-OFDM (solid lines) and NCMC-CDMA (dashed lines) transceiver.

and MC-CDMA symbol period, the BER results for an NCOFDM and an NC-MC-CDMA transceiver for different percentages of deactivated subcarriers are shown in Fig. 1(b). Generally, the performance of the NC-OFDM transceiver is better than that of the MC-CDMA transceivers. The mean BER performance for a frequency selective channel closely follows theoretical expectations as shown in Fig. 2(b) In particular, as the percentage of deactivated subcarriers increases, the BER performance of the NC-MC-CDMA system worsens while the BER performance of the NC-OFDM transceivers improves slightly.

Total throughput of a $\mathrm{N}$-subcarrier NC-OFDM system is $N R_{b}$. In NC-OFDM system, data is not transmitted over subcarriers corresponding to the spectrum occupied by the incumbent users, with prior knowledge of ISO. Therefore, there would be no information loss in NC-OFDM scheme. Moreover, SNR gain for increased ISO would result in improved BER performance of NC-OFDM scheme.

In NC-MC-CDMA system, input symbols are spreaded over the available bandwidth. Therefore, turning off 


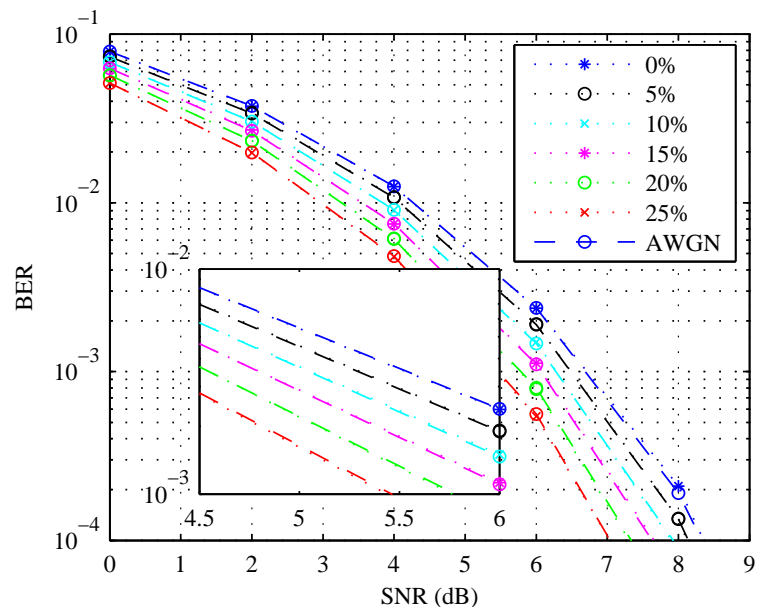

(a) AWGN channel.

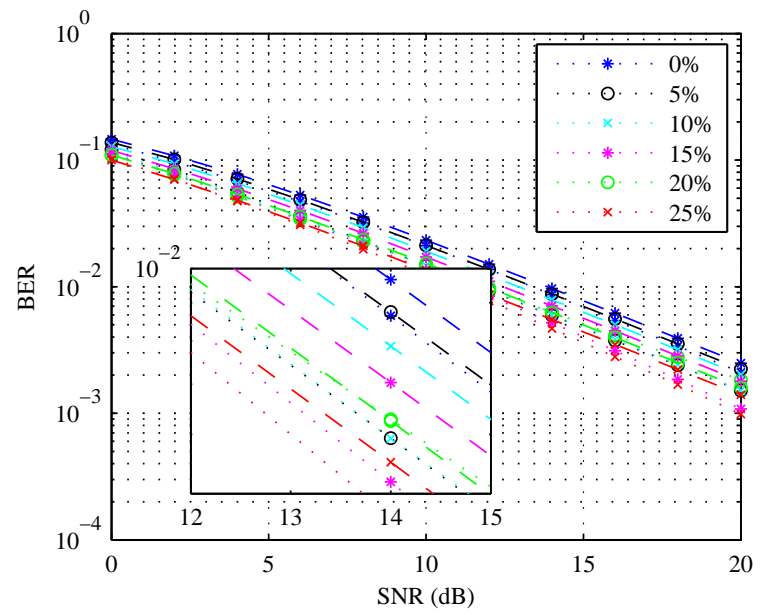

(b) 3-path Rayleigh channel.

Fig. 2 BER performance of the NC-OFDM transceiver. Note that the dashed lines represent the analytic results while the dotted lines are the results via simulation.

the subcarriers corresponding to incumbent user spectrum would result in fractional loss of information. This partial loss of information may also result in losing orthogonality between the spreading codes. Hence, NC-MC-CDMA BER performance degrades for higher ISO.

\section{Conclusion}

In this paper, we presented two candidates for agile modulation in cognitive radio transceivers operating in a DSA network. We evaluated and compared the error robustness of NC-OFDM and NC-MC-CDMA transceivers (both analytically and through simulations) operating in an AWGN and multipath channels and compared it with NC-MC-CDMA. From the SNR analysis, it is observed that BER performance of NC-OFDM is superior to the NC-MC-CDMA system, when the available transmission spectrum is noncontiguous.

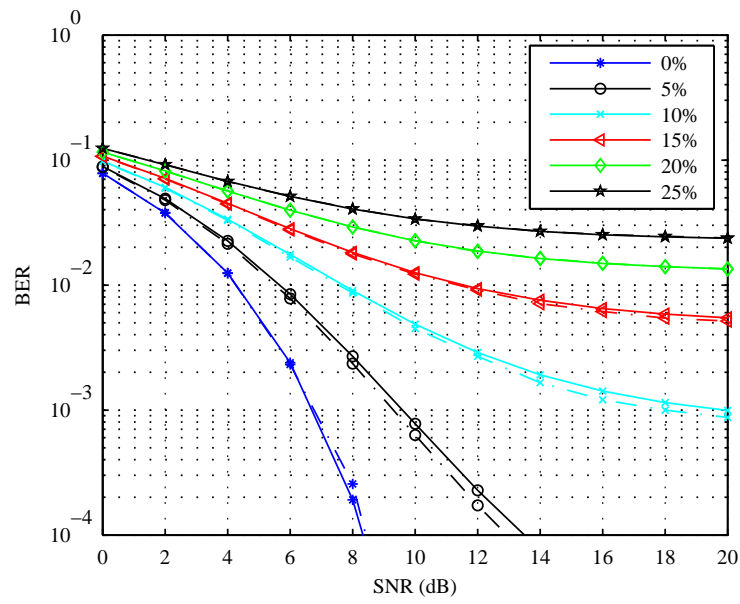

Fig. 3 Analytical (dashed line) and simulation (solid line) results of NC-MC-CDMA BER performance in AWGN channel.

\section{References}

[1] Federal Communications Commission, "Unlicensed operation in the TV broadcast bands." ET Docket No. 04-186, 2004.

[2] J. Mitola, III, "Cognitive radio for flexible mobile multimedia communications," in Proc. IEEE Int. Wksp. Mobile Multimedia Commun., vol. 1, (San Diego, CA, USA), pp. 3-10, Nov. 1999.

[3] R. Kimura and F. Adachi, "Comparison of OFDM and multicode MC-CDMA in frequency selective fading channel," Electron. Lett., vol. 39, pp. 317-318, Feb. 2003.

[4] B.-J. Choi, E.-L. Kuan, and L. Hanzo, "Crest-factor study of MCCDMA and OFDM," in Proc. 50th IEEE Veh. Technol. Conf. Fall, vol. 1, (Amsterdam, The Netherlands), pp. 233 - 237, Sept. 1999.

[5] R. Rajbanshi, Q. Chen, A. M. Wyglinski, J. B. Evans, and G. J. Minden, "Comparative study of frequency agile data transmission schemes for cognitive radio transceivers," in Proc. of 1st Int. Wksp. on Technol. and Policy for Accessing Spectrum, (Boston, MA, USA), Aug. 2006.

[6] T. A. Weiss and F. K. Jondral, "Spectrum pooling: an innovative strategy for the enhancement of spectrum efficiency," IEEE Commun. Mag., vol. 43, pp. S8 - 14, Mar. 2004.

[7] J. D. Poston and W. D. Horne, "Discontiguous OFDM considerations for dynamic spectrum access in idle TV channels," in Proc. IEEE Int. Symp. New Frontiers Dynamic Spectr. Access Networks, vol. 1, (Baltimore, MD, USA), pp. 607-610, Nov. 2005.

[8] B. R. Saltzberg, "Comparison of single-carrier and multitone digital modulation for ADSL applications," IEEE Commun. Mag., vol. 36, pp. 114-121, Nov. 1998.

[9] J. A. C. Bingham, "Multicarrier modulation for data transmission: An idea whose time has come," IEEE Commun. Mag., vol. 28, pp. 5-14, May 1990.

[10] R. Etkin, A. Parekh, and D. Tse, "Spectrum sharing for unlicensed bands," in Proc. IEEE Int. Symp. New Frontiers Dynamic Spectr. Access Networks, vol. 1, (Baltimore, MD, USA), pp. 251-258, Nov. 2005.

[11] F. Weidling, D. Datla, V. Petty, P. Krishnan, and G. J. Minden, "A framework for RF spectrum measurements and analysis," in Proc. IEEE Int. Symp. New Frontiers Dynamic Spectr. Access Networks, vol. 1, (Baltimore, MD, USA), pp. 573-576, Nov. 2005.

[12] L. Hanzo, M. Munster, B. J. Choi, and T. Keller, OFDM and MC-CDMA for Broadband Multi-user Communications, WLANs and Broadcasting. IEEE Press, 2003.

[13] J. G. Proakis, Digital Communications. New York, NY: McGraw Hill, 2001.

[14] T. S. Rappaport, Wireless Communications: Principles and Practice. Upper Saddle River, NJ, USA: Prentice Hall, 1996.

[15] K. S. Shanmugan and A. M. Breipohl, Random Signals: Detection, Estimation and Data Analysis. USA: John Wiley \& Sons, 1988. 